\title{
Diabetes, treatments for diabetes and their effect on cancer incidence and mortality: attempts to disentangle the web of associations
}

\author{
S. H. Wild
}

Received: 7 March 2011 / Accepted: 18 March 2011 /Published online: 4 May 2011

(C) Springer-Verlag 2011

\begin{abstract}
The association between type 2 diabetes and some types of cancer is well established and the complexity of this association is receiving increasing recognition. Many factors influence the risk of both diabetes and cancer including age, sex, ethnicity, socioeconomic status, obesity/insulin resistance, diet (including alcohol intake), physical activity levels and smoking history. The presence of diabetes may influence the uptake of cancer screening. Using an experimental approach to investigate the effect of better control of diabetes is helpful in assessing whether hyperglycaemia has a causal relationship with cancer. Diabetes treatments may influence the risk of cancer independently of their effect on glycaemia and complicate investigation of the association between diabetes and cancer. This article discusses the complexity involved in attempting to clarify the factors that contribute to the associations between diabetes, hyperglycaemia, diabetes treatment and cancer.
\end{abstract}

Keywords Epidemiology · Insulin therapy · Oral pharmacological agents

$\begin{array}{ll}\text { Abbreviations } \\ \text { ACCORD } & \begin{array}{l}\text { Action to Control Cardiovascular Risk in } \\ \text { Diabetes }\end{array} \\ \text { ADVANCE } & \begin{array}{l}\text { Action in Diabetes and Vascular Disease: } \\ \text { Preterax and Diamicron-MR Controlled } \\ \text { Evaluation }\end{array}\end{array}$

Diabetes is associated with increased risks of bladder, breast, colorectal, endometrial, kidney, liver and pancreatic cancer and a lower risk of prostate cancer compared with the

\section{S. H. Wild $(\square)$}

Reader in Epidemiology and Public Health, Centre for Population

Health Sciences, University of Edinburgh,

Teviot Place, Edinburgh EH8 9AG, UK

e-mail: sarah.wild@ed.ac.uk incidence of these cancers among people without diabetes or general populations [1]. These associations are modulated by obesity, or by the insulin resistance associated with obesity, both of which are commonly present in people with type 2 diabetes. Other potential confounding variables include, for example, altered exposure to the tissue effects of oestrogen or testosterone, low-grade inflammation, effects of diabetes therapies other than glucose-lowering and possibly differential uptake of cancer screening between people with and without diabetes. As a consequence, the extent to which hyperglycaemia - the defining characteristic of diabetes - is responsible for the increased prevalence of cancer is unclear.

The statistician Austin Bradford Hill set out useful criteria for identifying causality; these are summarised in the text box [2]. The relationship between diabetes and cancer risk is not strong - other than for liver and pancreatic cancer [1], which may occur as a consequence of reverse causality - with the cancers increasing risk of diabetes rather than diabetes increasing risk of the cancers. If hyperglycaemia were indeed a major influence on cancer development, one would anticipate consistent consequences from exposure to sustained hyperglycaemia for people with type 1 and type 2 diabetes. The risk of cancer in type 1 diabetes has been examined in a limited number of studies. A Swedish study identified people admitted to hospital with a record of type 1 diabetes between 1965 and 1999, excluded the first year of follow-up and used data linkage to compare cancer incidence in this group vs the whole population [3]. There were 355 incident cases of cancer among 29,187 people who had been admitted to hospital with a record of type 1 diabetes, with standardised incidence ratios of 1.2 (95\% CI 1.0-1.3) for all cancers, 2.3 (95\% CI 1.1-4.1) for stomach cancer, 1.6 (95\% CI 1.1-2.2) for cervical cancer and 2.7 (95\% CI 1.4-4.7) for endometrial cancer. The authors acknowledged the potential for chance findings and possible misclassification bias introduced by the inclusion of people with type 2 diabetes in the study population. A cohort study of 
insulin-treated patients in the UK who received a diagnosis of diabetes under 30 years of age between 1972 and 1986 identified 214 incident malignancies of any type (excluding non-melanoma skin cancer) among the 23,834 participants [4]. Of 17 site-specific malignancies that were considered, the only statistically significant association was with ovarian cancer, which developed in 16 women in the study population. Incidence ratios standardised for age, sex, calendar year and country were 0.95 (95\% CI $0.84-1.08)$ and 2.14 (95\% CI 1.22-3.48) for all cancers and ovarian cancer, respectively. A systematic review and meta-analysis of three cohort and six case-control studies in which 39 occurrences of pancreatic cancer were identified reported a relative risk for pancreatic cancer among people with type 1 and young-onset diabetes compared with those without diabetes of $2.00(95 \%$ CI 1.37-3.01).[5]. It is of note that there is limited overlap between the cancer types identified in these studies and those identified as being associated with type 2 diabetes. Although these studies, therefore, do not provide convincing evidence of a consistent association between hyperglycaemia and cancer, it is biologically plausible that hyperglycaemia might promote tumour growth, since cancer cells require glucose for aerobic glycolysis (the Warburg effect) [6]. Consistent with this hypothesis and the criterion for a dose-response relationship or biological gradient, $\mathrm{HbA}_{1 \mathrm{c}}$ values $>6.5 \%$ are linearly associated with an increased risk of cancer, although this relationship is unlikely to be independent of insulin resistance, obesity and diabetes therapy [7].

\section{Bradford Hill criteria for supporting a causal association between an exposure/ risk}

\section{factor and an outcome [2]}

- Strength A small association does not mean that there is not a causal effect, though the larger the association, the more likely that it is causal

- Consistency Consistent findings observed in different populations strengthen the likelihood of an effect

- Specificity The more specific an association between an exposure and an outcome is, the greater the probability of a causal relationship

- Temporality The outcome has to occur after the exposure (and if there is an expected delay between the exposure and outcome, then the outcome must occur after that delay)

- Biological gradient Greater exposure should generally lead to higher risk of the outcome (or lower risk if the exposure is protective). However, in some cases, the mere presence of the factor can trigger the effect

- $\quad$ Plausibility A plausible mechanism between exposure and outcome provides support but is limited by current knowledge of biological mechanisms

- Coherence Coherence between epidemiological and laboratory findings increases the likelihood of a causal relationship. However, Hill noted that '... lack of such [laboratory] evidence cannot nullify the epidemiological effect on associations'

- Experiment Support for a causal relationship if provided if adding or removing the exposure in a controlled experiment influences the outcome

- Analogy The presence of associations between other similar exposures/outcomes supports causality 
A further criterion for causality is the ability of experimental intervention to alter exposure levels and outcome. Randomised controlled trials of intensified glucose control provide control of confounding by non-treatment factors and baseline allocation bias (a term indicating that, outside the setting of a randomised controlled trial, people who receive a specific treatment differ from those who do not receive the treatment in characteristics that influence the outcome). The difference in the findings of observational studies and randomised controlled trials is illustrated by recent metaanalyses. The meta-analysis of observational studies suggests a protective effect against cancer with a relative risk for metformin use compared with other glucose-lowering drugs with a relative risk (RR) for all cancers combined of 0.69 (95\% CI 0.61-0.79) [8]. In contrast, a preliminary metaanalysis of trials has reported a modest, non-statistically significant positive association between randomisation to metformin compared with other treatments and the incidence of all cancers (RR 1.10, 95\% CI 0.88-1.37) [9].

No differences in cancer incidence between the intensive and standard treatment groups have been reported from any of the four major trials of intensified glucose control (the UK Prospective Diabetes Study [UKPDS, all arms], the Veterans Affairs Diabetes Trial [VADT], Action to Control Cardiovascular Risk in Diabetes and Action in Diabetes [ACCORD] and Vascular Disease: Preterax and DiamicronMR Controlled Evaluation [ADVANCE]) [10]. A metaanalysis has included data from the above studies and from trials resulting in statistically significant and clinically important differences in glycaemic control between treatment arms, namely, the Prospective Pioglitazone Clinical Trial in Macrovascular Events (PROactive) and Rosiglitazone Evaluated for Cardiac Outcomes and Regulation of Glycemia in Diabetes (RECORD) trials. Again, there was no association between groups in terms of cancer mortality (RR 1.00, 95\% CI 0.81-1.24) or cancer incidence (RR 0.91, 95\% CI 0.79-1.05) [11]. The meta-analysis reported outcomes for all cancers combined which could conceal differing effects of glucose control on different cancers but further data are needed to test this hypothesis.

This edition of Diabetologia reports more detailed results on cancer risk over a median 5 year follow-up in the ADVANCE trial, in which a lower mean $\mathrm{HbA}_{1 \mathrm{c}}$ at follow-up (a $0.7 \%$ decrement from a mean of $7.5 \%$ at baseline) was achieved in the intensive control group compared with the standard control group [12]. Data from an earlier paper describing the effect of the intervention on vascular outcomes, which included numbers of hospital admissions for neoplasms of any type by treatment group (119 in each group) [13], were included in the metaanalysis by Johnson and Bowker [11]. The current study extends the previous report, and includes 328 and 303 solid cancer outcomes (excluding neoplasms of lymphoid tissue) in the intensive and standard glycaemic control groups, respectively. The hazard ratio for incidence of a solid cancer was 1.09 (95\% CI 0.93-1.27) for intensive vs standard glycaemic control, suggesting a modest, nonstatistically significant positive association with all cancers combined, although the authors raise the possibility of ascertainment bias in the intensive treatment group. Outcome data were based on serious adverse reports and were not routinely validated. There were 41 and 35 cancer deaths in the intensive and standard treatment groups, respectively (hazard ratio 1.17 , 95\% CI $0.75-1.84$ ). The authors found no statistically significant evidence of a difference in incidence of specific cancers or groups of cancers between treatment groups. They acknowledge the limited power of their study to detect associations with specific cancers as, for example, there were only 64 breast cancers and 32 pancreatic cancers identified in the study. The authors conclude that the findings of their study do not support the hypothesis that intensification of glucose control reduces the risk of cancer incidence and mortality.

The pattern of therapeutic intervention in trials of intensified glucose control is complex, and involves multiple agents. Intensive control groups are exposed to more diabetes treatments than standard control groups. The authors of this paper, quite appropriately, did not attempt to investigate the effects of individual drugs on the outcome using the existing study design. People whose diabetes can be controlled with fewer drugs are likely to differ from those who require more drugs in ways that may influence risk of cancer. Performing analyses by treatment group would destroy the effects of randomisation and can introduce bias and confounding. Investigation of the effect of individual drugs requires further analyses of the individual and time-varying exposure to each drug or class of drugs [11].

Since hyperglycaemia, as such, does not appear to play a major role in defining susceptibility to cancer, the role of the individual drugs used to treat diabetes comes into greater prominence. The potential effects of different treatments also need to be considered in the context of diabetes aetiology, duration of disease, residual beta cell function and insulin sensitivity. Lifestyle or pharmacological interventions that affect insulin sensitivity, either directly or by affecting body weight, may influence cancer risk, independently of their effect on hyperglycaemia. Weight loss following bariatric surgery has been shown to be associated with reduced cancer incidence and mortality in women participating in the Swedish Obese Subjects Study [14, 15] and cancer mortality in both men and women in a US study [16]. These findings suggest that weight loss (presumably resulting in improved insulin sensitivity) reduces subsequent risk of cancer. Furthermore, potential benefits in terms of glycaemic control obtained with some treatments 
may be counter-balanced by increasing levels of obesity and insulin resistance. Participants in the intensive control groups of the various trials gained more weight on average than the standard control groups [13, 17], although for ADVANCE participants mean weight at follow-up was only $0.7 \mathrm{~kg}$ greater in the intensive compared with the standard control group [13]. There was a $3.1 \mathrm{~kg}$ difference in weight gain between groups among ACCORD participants between baseline and follow-up, with $28 \%$ of participants gaining more than $10 \mathrm{~kg}$ [17].

The available evidence therefore does not provide convincing support for the hypothesis that hyperglycaemia itself influences cancer risk. However, obesity and insulin resistance appear to confound the association with cancer observed in people with type 2 diabetes. The additional effects of treatments for diabetes and the factors underlying the need for such treatment complicate the study of the association between diabetes and cancer further. Several previous commentaries have identified the difficulties of interpreting the results of observational studies of the association between diabetes treatment and risk of cancer $[18,19]$. The effect of allocation bias is likely to contribute to any apparent associations. It is not feasible to investigate cancer as the primary outcome in trials of diabetes treatments other than short-term studies of first-line agents given the patterns of disease progression among people with diabetes and the requirement for large sample sizes and long-term follow-up. However, as described above and in a recent consensus report [20], the appropriate interpretation of well-designed, appropriately powered observational studies and trials can provide a valuable contribution to attempts to elucidate effects of hyperglycaemia, diabetes and its different treatments on cancer risk.

Duality of interest $S$. Wild has received an honorarium for participating in a Novo Nordisk advisory board.

\section{References}

1. Vigneri P, Frasca F, Sciacca L, Pandini G, Vigneri R (2009) Diabetes and cancer. Endocr Relat Cancer 16:1103-1123
2. Hill AB (1965) The environment and disease: association or causation? Proc R Soc Med 58:295-300

3. Zendehdel K, Nyren O, Ostenson CG, Adami HO, Ekbom A, Ye W (2003) Cancer incidence in patients with type 1 diabetes mellitus: a population-based cohort study in Sweden. J Natl Cancer Inst 95:1797-1800

4. Swerdlow AJ, Laing SP, Qiao Z et al (2005) Cancer incidence and mortality in patients with insulin-treated diabetes: a UK cohort study. Br J Cancer 92:2070-2075

5. Stevens RJ, Roddam AW, Beral V (2007) Pancreatic cancer in type 1 and young-onset diabetes: systematic review and metaanalysis. Br J Cancer 96:507-509

6. Warburg O (1956) On the origin of cancer cells. Science 123:309314

7. Yang X, Ko GT, So WY et al (2010) Associations of hyperglycemia and insulin usage with the risk of cancer in type 2 diabetes: the Hong Kong diabetes registry. Diabetes 59:1254-1260

8. DeCensi A, Puntoni M, Goodwin P et al (2010) Metformin and cancer risk in diabetic patients: a systematic review and metaanalysis. Cancer Prev Res (Phila) 3:1451-1461

9. Stevens RJ, McLellan J, Cairns BJ et al (2011) Cancer outcomes in randomised clinical trials of metformin: the Metformin Trialists' Collaboration. Diabet Med 28:193 (Abstr.)

10. Gerstein HC (2010) Does insulin therapy promote, reduce, or have a neutral effect on cancers? JAMA 303:446-447

11. Johnson JA, Bowker SL (2011) Intensive glycaemic control and cancer risk in type 2 diabetes: a meta-analysis of major trials. Diabetologia 54:25-31

12. Stefansdottir G, Zoungas $S$, Chalmers $J$ et al (2011) Intensive glucose control and risk of cancer in patients with type 2 diabetes. Diabetologia. doi:10.1007/s00125-011-2104-x

13. Patel A, MacMahon S, Chalmers J et al (2008) Intensive blood glucose control and vascular outcomes in patients with type 2 diabetes. N Engl J Med 358:2560-2572

14. Sjostrom L, Gummesson A, Sjostrom CD et al (2009) Effects of bariatric surgery on cancer incidence in obese patients in Sweden (Swedish Obese Subjects Study): a prospective, controlled intervention trial. Lancet Oncol 10:653-662

15. Sjostrom L, Narbro K, Sjostrom CD et al (2007) Effects of bariatric surgery on mortality in Swedish obese subjects. N Engl J Med 357:741-752

16. Adams TD, Gress RE, Smith SC et al (2007) Long-term mortality after gastric bypass surgery. N Engl J Med 357:753-761

17. Gerstein HC, Miller ME, Byington RP et al (2008) Effects of intensive glucose lowering in type 2 diabetes. N Engl J Med 358:2545-2559

18. Pocock SJ, Smeeth L (2009) Insulin glargine and malignancy: an unwarranted alarm. Lancet 374:511-513

19. Smith U, Gale EA (2010) Cancer and diabetes: are we ready for prime time? Diabetologia 53:1541-1544

20. Giovannucci E, Harlan DM, Archer MC et al (2010) Diabetes and cancer: a consensus report. Diabetes Care 33:1674-1685 